\title{
Enhanced cellular uptake and long-term retention of chitosan-modified iron-oxide nanoparticles for MRI-based cell tracking
}

This article was published in the following Dove Press journal:

International Journal of Nanomedicine

2I August 2012

Number of times this article has been viewed

\author{
Sasha H Bakhru' \\ Eda Altiok ${ }^{2}$ \\ Christopher Highley' \\ Daniel Delubac' \\ Joseph Suhan ${ }^{2}$ \\ T Kevin Hitchens ${ }^{2}$ \\ Chien $\mathrm{Ho}^{2}$ \\ Stefan Zappe'
}

'Department of Biomedical

Engineering, ${ }^{2}$ Department of Biological

Sciences, Carnegie Mellon University,

Pittsburgh, PA, USA
Correspondence: Stefan Zappe

Department of Biomedical Engineering,

Carnegie Mellon University,

PTC 4319, 700 Technology Drive,

Pittsburgh, PA I5219, USA

$\mathrm{Tel}+\mathrm{I} 4127361683$

Fax + I 4126874246

Email zappe@cmu.edu
Abstract: Tracking cells after therapeutic transplantation is imperative for evaluation of implanted cell fate and function. In this study, ultrasmall superparamagnetic iron oxide nanoparticles (USPIO NPs) were surface functionalized with water-soluble chitosan, a cationic polysaccharide that mediates enhanced endocytic uptake, endosomal escape into the cytosol, and subsequent long-term retention of nanoparticles. NP surface and chitosan were independently fluorescently labeled. Our NPs enable NP trafficking studies and determination of fate beyond uptake by fluorescence microscopy as well as tracking of labeled cells as localized regions of hypointensity in $\mathrm{T}_{2}{ }^{*}$-weighted magnetic resonance imaging (MRI) images. Adult rat neural stem cells (NSCs) were labeled with NPs, and assessment of NSC proliferation rates and differentiation potential revealed no significant differences between labeled and unlabeled NSCs. Significantly enhanced uptake of chitosan NPs in comparison to native NPs was confirmed by transmission electron microscopy, nuclear magnetic resonance (NMR) spectroscopy and in vitro cellular MRI at 11.7 Tesla. While only negligible fractions of native NPs enter cells, chitosan NPs appear within membranous vesicles within 2 hours of exposure. Additionally, chitosanfunctionalized NPs escaped from membrane-bound vesicles within days, circumventing NP endo-lysosomal trafficking and exocytosis and hence enabling long-term tracking of labeled cells. Finally, our labeling strategy does not contain any NSC-specific reagents. To demonstrate general applicability across a variety of primary and immortalized cell types, embryonic mouse NSCs, mouse embryonic stem cells, HEK 293 kidney cells, and HeLa cervical cancer cells were additionally exposed to chitosan-USPIO NPs and exhibited similarly efficient loading as verified by NMR relaxometry. Our efficient and versatile labeling technology can support cell tracking with close to single cell resolution by MRI in vitro, for example, in complex tissue models not optically accessible by confocal or multi-photon fluorescence microscopy, and potentially in vivo, for example, in animal models of human disease or injury.

Keywords: nanoparticle, iron oxide, chitosan, neural stem cell, cell tracking

With the emergence of cell-based approaches to medical therapies spanning enzyme replacement and stem cell-based tissue repair, the ability to track living cells has proven imperative. For example, neural stem cells (NSCs) - multipotent neural precursors of the brain, capable of self renewing, homing, and differentiating into neurons and glia $^{1-3}$ - have opened up new avenues toward neural cell replacement therapies for neurodegenerative disease since their discovery within the mammalian brain. ${ }^{4-6}$ Recently, several groups have reported on progress toward treatment of diseases and disorders, including multiple sclerosis, ${ }^{7,8}$ Parkinson's disease, ${ }^{9,10}$ and traumatic central nervous system injury ${ }^{11,12}$ with transplanted NSCs. However, data from these animal trials and almost all others have been collected after necropsy of the recipient animals, ie, there is no data available 
regarding the route or rate of migration of cells during the first critical days after implantation. For example, Pastori et al recently observed through in vitro experimentation with isolated brains and subsequent immunohistochemical analysis of brain slices that arterially perfused NSCs migrated to but did not breach the barrier of ischemic injury in a disease model for stroke. ${ }^{13}$ In addition to such short-term experimentation in vitro, magnetic labeling of NSCs and tracking by magnetic resonance imaging (MRI) could yield valuable, long-term, in vivo data for example on when and how the migrating NSCs arrive at the site of ischemic injury and what behavior causes their arrested migration before settling into the adjacent injured tissue. Long-term tracking of transplanted NSCs will open entirely new avenues to studying disease mechanisms and developing therapeutic approaches.

Labeling cells with a sufficiently high intracellular density of ultrasmall superparamagentic iron oxide nanoparticles (USPIO NPs) with superior relaxivity ${ }^{14,15}$ (192.0 $\mathrm{mM}^{-1}$ $[\mathrm{Fe}] \mathrm{s}^{-1}$, from Supplementary Figure 1) enables NSC tracking as localized regions of signal hypointensity in $\mathrm{T}_{2} *$-weighted MRI images. Iron oxide generates large local magnetic field gradients that generate a phase incoherence of the surrounding signal. This "blooming effect" enables single cells to be detected as easily identifiable, relatively larger regions of signal hypointensity at resolutions beneath the single-cell scale; the effect can be up to 50-fold larger than the iron deposit or tens to hundreds of microns. The challenge, however, remains to efficiently induce cellular uptake and long-term cellular retention of paramagnetic particles by cells.

In previous studies superparamagnetic iron-oxide nanoparticles (SPIO) were used to label macrophages, enabling cellular MRI-based identification of organ rejection in animal models. ${ }^{15-18}$ Conveniently, macrophages phagocytose micronsized particles from their surroundings, and so labeling of these particular cells proved straightforward. Immortalized cell lines have been successfully labeled using synthetic transfection reagents, such as lipofectamine ${ }^{19}$ and polyethylene imine (PEI), ${ }^{20}$ however, such reagents are unacceptably cytotoxic at even low concentrations to a variety of primary somatic and stem cell types, limiting their utility for labeling of such cells. Surface conjugation of small molecules, such as cholera toxin B, and biologically active oligopeptides, such as arginineglycine-aspartic acid (RGD), to nanoparticles has also yielded high rates of endocytic cellular uptake, ${ }^{21,22}$ however, trafficking through endo-lysosomal pathways beyond uptake causes these particles to be rapidly evacuated from cells. ${ }^{21}$ Furthermore, engagement of specific cell surface receptors by these active molecules, ie, surface gangliosides by cholera toxin $\mathrm{B}^{21}$ and a dozen known integrins by RGD ${ }^{23}$ not only enables cell labeling but also effects cell fate, which may be undesirable for the mechanistic studies such labeling is meant to support.

Here, we present a potentially universal method for efficient magnetic labeling of mammalian cells utilizing the naturally derived, biocompatible, polyelectrolyte chitosan. Previously, complexation of chitosan and plasmid DNA to form chitosan/ DNA nanoparticle gene carriers was employed to achieve efficient cellular transfection, both in vitro and in vivo, as described in a seminal publication from Kam Leong and colleagues. By inducing primarily clathrin-mediated endosomal uptake of these NPs and subsequent charge-induced endosomal escape intracellulary, nuclear uptake of delivered DNA by proliferating cells and subsequent expression of delivered genes was made possible. ${ }^{24-26}$ Our methods for magnetic cell labeling based on chitosan-conjugated iron-oxide nanoparticles is similarly characterized by rapid endosomal nanoparticle uptake, subsequent endosomal escape, and corresponding long-term cytosolic retention. Our approach in particular is to surface functionalize ultrasmall $(\sim 5 \mathrm{~nm})$ SPIO NPs with water soluble chitosan - deacetylated chitin from crustacean shells. Chitosan is a linear polysaccharide of $\beta$-(1-4)-linked D-glucosamine (deacetylated) and N-acetyl-D-glucosamine that is polycationic ${ }^{27,28}$ and spontaneously deposits on the negatively charged carboxylated surface of a USPIO NP. This physical association is then strengthened by chemical crosslinking using carbodiimide chemistry to link primary amines on the chitosan backbone to carboxyl groups on the nanoparticle surface via an amide linkage. This surface modification with positively charged chitosan promotes nanoparticle attachment to negatively charged domains on the outer leaflet of the cell membrane, enhancing nanoparticle uptake by endocytosis. ${ }^{25,26}$ We applied this method to labeling and tracking of neural stem cells (a cell type that is both of tremendous therapeutic potential and particularly difficult to label without negatively effecting viability or function) as well as four other mammalian cell types, indicating general applicability of our approach.

\section{Methods}

\section{Chitosan-USPIO NP synthesis}

Aminated-USPIO surface conjugation with AlexaFluor 555

In order to attach the amine-reactive AlexaFluor 555 dye to the carboxylated surface of our nanopaticles (USPIO, Ocean Nanotech), a Schmidt reaction-driven conversion of $\sim 5 \%$ of the NP surface carboxylic acid groups to primary amines was undertaken by reacting carboxyl groups with sodium azide in an aqueous sulfuric acid solution. The surface-aminated 
particles were then combined with a molar excess of aminereactive dye, yielding nanoparticles of uniform, controllable, surface fluorescence. In detail, $0.5 \mathrm{~mL}$ of partially aminated NPs at $3.5 \mathrm{mg} / \mathrm{mL}$ with $1 \mu \mathrm{L}$ of $1 \mathrm{mg} / 10 \mu \mathrm{L}$ AlexaFluor 555 dye in DMSO was incubated at room temperature overnight. The unlabeled fluorescent dye and DMSO were removed by filtration using a $300-\mathrm{kDa}$ spin column, centrifuged for 5 minutes at $3000 \mathrm{rpm}$. The labeled NP suspension was then washed similarly in a spin column with phosphate-buffered saline (PBS) two more times, and NPs were recovered by gentle pipetting in PBS followed by transfer into a $1.5-\mathrm{mL}$ Eppendorf tube for storage.

\section{Chitosan conjugation with AlexaFluor 633}

Chitosan was labeled with a targeted ratio of about five fluorescent molecules per water-soluble chitosan-glutamate salt molecule (NovaMatrix), leaving a majority of primary amines free for surface complexation of chitosan with the negatively charged, similarly fluorescently labeled, nanoparticle surface. In detail, $0.7 \mathrm{~mL}$ of chitosan stock $(1 \mathrm{wt} / \mathrm{vol} \%$ Protosan UPG213, 40,000-200,000 molecular weight [MW], Pronova Biopolymer) was incubated with $1 \mu \mathrm{L}$ of $1 \mu \mathrm{g} / \mu \mathrm{L}$ AlexaFluor 633 dye in DMSO stock solution at room temperature overnight. The solution was then dialyzed (14 kDa MWCO Pierce Snakeskin ${ }^{\circledR}$ membrane) against running deionized water for 24 hours, then transferred from the dialysis membrane to $50 \mathrm{~mL}$ Falcon tubes and flash frozen in liquid nitrogen. The fluorescently labeled chitosan was then recovered by lyophilization until a dry fibrous solid was achieved.

\section{Carbodiimide-based conjugation of USPIO NPs and chitosan}

The targeted molar ratio of chitosan molecules to carboxylated iron oxide NPs chosen was 5:1 such that each nanoparticle should be surface conjugated to approximately five chitosan molecules, affording a high degree of surface coverage. In order to achieve this ratio with a $175,000-\mathrm{kDa}$ chitosan glutamate salt and $5 \mathrm{~nm}$ NPs, $0.2 \mathrm{~mL}$ of AlexaFluor 555-labeled NP stock (at $3.5 \times 10^{-9} \mathrm{~mol} \mathrm{NPs}$ ) was conjugated to $0.7 \mathrm{~mL}$ AlexaFluor 633 -labeled chitosan stock $\left(1.75 \times 10^{-8} \mathrm{~mol}\right.$ chitosan) via standard carbodiimide chemistry to conjugate carboxylic acid groups on the NP surface with randomly distributed primary amines on D-glucosamine residues along the chitosan backbone. Stock solutions of $25 \mathrm{mg} / \mathrm{mL}$ each of EDC (Pierce) and sulfo-NHS (Thermo Scientific) were prepared in $0.1 \mathrm{M}$ MES buffer prior to conjugation. To prepare the carboxylated nanoparticles for amine conjugation, $16 \mu \mathrm{L}$ of EDC stock and $44 \mu \mathrm{l}$ of sulfo-NHS stock were combined in $0.2 \mathrm{~mL}$ of NP suspension. The mixture was incubated for 30 minutes at room temperature, rocking gently on an orbital shaker. Then, $0.7 \mathrm{~mL}$ of dissolved chitosan stock was added to the nanoparticle suspension, and the solution was placed again on an orbital shaker and the reaction allowed to proceed for 2 hours at room temperature. Free chitosan and other reagents were removed from the nanoparticles by centrifugation in spin columns ( $300 \mathrm{kDa}$, Pierce) for 5 minutes at $3000 \mathrm{rpm}$ in a benchtop microcentrifuge (Eppendorf). The flow through was discarded, and the retained nanoparticles were washed within the spin columns $5 \times$ with PBS. Finally, the total resuspension volume of PBS was increased to a total of $500 \mu \mathrm{L}$ per column. The suspensions from the spin columns were then combined and stored at $4^{\circ} \mathrm{C}$.

\section{NSC culture and labeling with USPIO NPs}

Adult rat NSCs were cultivated in neurobasal media with B27 supplement (Chemicon) with $20 \mathrm{ng} / \mathrm{mL}$ of FGF-2 (Invitrogen) for phenotype maintenance; NSCs were seeded on poly-L-onithine/laminin-coated tissue culture polystyrene (TCPS) vessels (either T25 flasks for expansion or 24-well plates for experimentation). Every third day, $50 \%$ of culture media was replaced with fresh media. For performance of differentiation experiments, replacement media was no longer supplemented with FGF-2. For experimentation, NSCs were trypsinized and centrifuged at $300 \mathrm{~g}$ for 5 minutes to produce a stable cell pellet, and supernatant media was aspirated away. Cells were washed once with PBS, followed by vigorous pipetting to break up any cell clusters. NSCs were centrifuged again, supernatant PBS discarded, and NSCs resuspended in $1 \mathrm{~mL}$ PBS at $5 \times 10^{4}$ cells $/ \mathrm{mL}$ and with $50 \mu \mathrm{L}$ of the chitosan-USPIO NP stock solution added to the cells (for a concentration of $30 \mu \mathrm{g} / \mathrm{mL}$, amount of chitosan-USPIO NP solution was adjusted for other loading concentrations). NSCs were then incubated for 2 hours in suspension within the same centrifuge tube at $37^{\circ} \mathrm{C}$ in a sterile culture incubator. NSCs were then centrifuged again and washed twice with PBS, centrifuged between washes to remove as many nanoparticles remaining in the supernatant PBS as possible. Supernatant fluid was then removed after a final washing step, and labeled NSCs were resuspended in complete media and cultured in poly-L-ornithine/lamin-coated flasks.

\section{Transmission electron microscopy sample preparation}

Roughly 1 million NSCs were prepared for each imaged sample as described above for cells treated with chitosan-USPIO NP 
or unmodified NP solution at $0,7.5,15,30$, and $60 \mu \mathrm{g} / \mathrm{mL}$ loading concentrations for 30 minutes each. NSCs were then prepared for transmission electron microscopy (TEM) by fixation with $2 \%$ glutaraldehyde in PBS. Fixed cells were washed three times with PBS and then treated with $1 \% \mathrm{OsO}_{4}$ in $\mathrm{PBS}$ for contrast enhancement of cellular features. Cells were then washed with distilled water and dehydrated using increasing concentrations of ethanol. Cells were then infiltrated overnight with a 1:1 mixture of epon-araldite and propylene oxide, desiccated, sectioned (100 nm) with a DDK diamond knife on a Reichert-Jung Ultracut E ultramicrotome, and stained with uranyl acetate and lead citrate for contrast enhancement. A Hitachi 7100 transmission electron microscope operating at $75 \mathrm{keV}$ coupled with an AMT Advantage 10 CCD camera system and National Institutes of Health image software was used to acquire images of the sections.

\section{Preparation of NSCs for nuclear magnetic resonance spectroscopy and $M R I$ imaging}

NSCs treated with chitosan-USPIO NP or unmodified NP solution at $0,7.5,15,30$, and $60 \mu \mathrm{g} / \mathrm{mL}$ loading concentrations were prepared as described above and seeded at $5 \times 10^{5} \mathrm{NSCs} / \mathrm{mL}$ in a $2 \mathrm{wt} / \mathrm{vol} \%$ ultra-low melting temperature agarose-in-PBS solution (SeaPrep Agarose; New England Biotech) to mimic in vitro the water-rich environment within brain tissue. Cell suspensions were transferred to 5-mm diameter nuclear magnetic resonance (NMR) tubes (for relaxometry) or to $1.5-\mathrm{mm}$ diameter glass capillary tubes (for MRI imaging) and incubated for $30 \mathrm{~min}$ at room temperature to gel the agarose prior to imaging. NSCs in NMR tubes were subjected to relaxometry measurements using a Bruker Minispec $(0.47 \mathrm{~T})$ system to measure the transverse relaxation time $\left(\mathrm{T}_{2}\right)$. NSCs in capillary tubes were subsequently imaged at high resolution using a Bruker Avance DRX 11.7 Tesla microimaging system equipped with a four-turn transverse RF coil, using a T2*-weighted gradient echo. The following parameters were used: TR $($ repetition time $)=1000 \mathrm{~ms}, \mathrm{TE}($ echo time $)=6 \mathrm{~ms}$, slice thickness $=100 \mu \mathrm{m}$, field of view $=2.5 \mathrm{~mm}$, and in-plane resolution $=39 \mu \mathrm{m}(64 \times 64$ matrix $)$.

\section{Immunocytochemical staining of cells}

Supernatant media was aspirated away from cells in culture. Cells were then washed twice with PBS, fixed in $4 \%$ paraformaldehyde (PFA; Electron Microscopy Sciences, Hatfield, PA) in PBS at room temperature for 45 minutes and simultaneously blocked and permeabilized using
Tris-buffered saline (TBS) with 5\% donkey serum (Jackson Immunoresearch, West Grove, PA) and $0.25 \%$ Triton X-100 (Sigma) for 60 minutes. Primary antibody solutions were prepared in TBS with Triton X-100 but without serum (TBS+). Blocking/permeabilization solution was aspirated away and replaced with primary antibody solution and allowed to incubate overnight at $4^{\circ} \mathrm{C}$. Primary antibody solution was aspirated away and samples washed $3 \times$ with TBS+. Secondary antibodies were prepared similarly in TBS+ and incubated for 60 minutes at room temperature. Samples were then washed again $3 \times$ with TBS+, and the nuclei stained with DAPI. Primary antibodies were used against MAP2 (neuronal marker, rabbit, Abcam, 1:500 dilution), GFAP (astrocyte marker, mouse, Abcam, 1:1000 dilution), and Nestin (NSC marker, rabbit, Abcam, 1:1000 dilution). Corresponding secondary antibodies were used to label the primary antibodies - DyLight 488-conjugated goat anti-rabbit immunoglobulin (Ig)G antibody (Jackson Immunoresearch, 1:250 dilution) and DyLight 488-conjugated goat anti-mouse IgG antibody (Jackson Immunoresearch, 1:250 dilution). Fluorescence imaging was performed on a Leica TCS SP5 confocal fluorescence microscope.

\section{Results and discussion}

Chitosan-USPIO NPs were synthesized as described above and shown in Figure 1A, with the chitosan and nanoparticle surfaces independently fluorescently labeled with amine

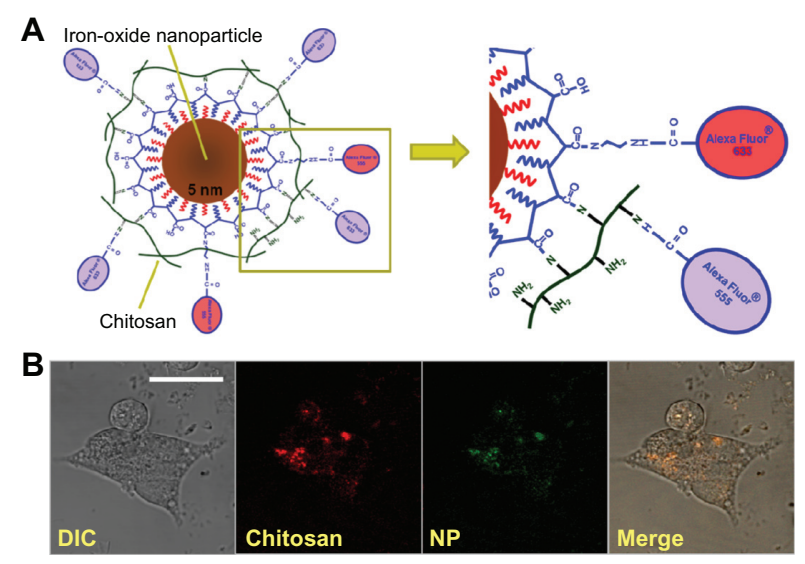

Figure I Chitosan-USPIO NP design and confocal fluorescence imaging of labeled cells. (A) Schematic diagram of chitosan/iron oxide nanoparticle conjugate (chitosan-USPIO NP). The fractionally aminated nanoparticle surface is labeled with an amine reactive AlexaFluor 633 fluorescent dye. Chitosan is similarly labeled with an Alexa Fluor ${ }^{\circledR} 555$ dye and conjugated to the nanoparticle surface via carbodiimide chemistry. (B) DIC and confocal fluorescence micrographs of fluorescent chitosanUSPIO NPs loaded at low concentration $(I \mu \mathrm{g} / \mathrm{mL})$ into NSCs in adherent culture on laminin-coated coverslips.

Note: The two fluorescent channels overlay and the particles appear to cluster within the cell, outside of the nucleus, 2 hours after cell labeling (scale bar $50 \mu \mathrm{m}$ ). Abbreviations: USPIO NPs, ultrasmall superparamagnetic iron oxide nanoparticles; DIC, Differential Interference Contrast; NSCs, neural stem cells. 
reactive AlexaFluor 555 and 633 carboxylic acid succinimidyl esters (Invitrogen), respectively, for multicolor tracking of uptaken particles. The apparent sizes and zeta potentials of the constituents and chitosan-USPIO NPs, as measured by dynamic light scattering, are given in Supplementary Figure 2, yielding a final apparent NP diameter of roughly $120 \mathrm{~nm}$, well within the range of permissable sizes for endocytic uptake. Double fluorescent labeling of chitosan and NP surface enables fluorescence-based tracking of each component independently to study intracellular chitosanUSPIO NP trafficking with time. For example, within the cell, chitosan may be enzymatically cleaved from the particle surface, and so beyond uptake, chitosan and NPs may be trafficked dissimilarly.

Confocal microscopy initially revealed the presence of chitosan-USPIO NPs within NSCs incubated with these particles, with overlapping fluorescence emission for the labeled chitosan and NPs at the earliest time points (Figure 1B). For a more detailed analysis over time, 2 hours after labeling, each labeled component was simultaneously but independently subjected to tracking by time-lapse, confocal, fluorescence imaging, with quantification of integrated mean fluorescence intensity within a single focal plane within cells (Supplementary Figure 3). These measurements showed that USPIO NP mean fluorescence intensity changed markedly over small time scales (Supplementary Figure 3, bottom graph), suggesting the movement of fluorescent packets into and out of the plane of imaging (ie, nanoparticles in membranous vesicles). In contrast, chitosan fluorescence intensity (Supplementary Figure 3, top graph) did not display as much variability over small time scales, suggesting that chitosan is present in a more distributed way inside and, more importantly, also outside of vesicles, with some chitosan detaching from the NP surface and escaping from membranous vesicles over hours. Detectable chitosan escape seems to foreshadow NP endosomal escape, with some chitosan making its way out of the vesicle at early time points but not enough to hinder later chitosan-USPIO NP escape, as discussed in detail further below.

In order to characterize further chitosan-USPIO NP uptake in comparison to native USPIO NP uptake, TEM imaging, NMR relaxometry, and MRI imaging were applied. NSCs were treated with chitosan-modified and unmodified NPs over a range of concentrations spanning $0-60 \mu \mathrm{g} / \mathrm{mL}$. TEM revealed
A
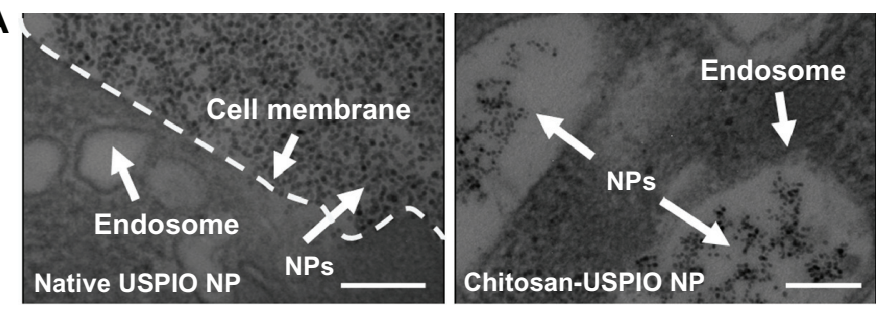

B

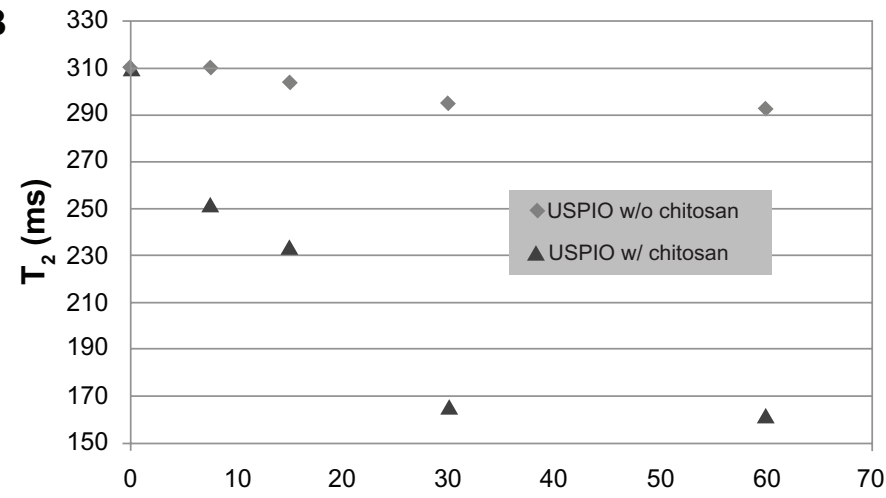

USPIO NP loading concentration $(\mu \mathrm{g} / \mathrm{mL})$

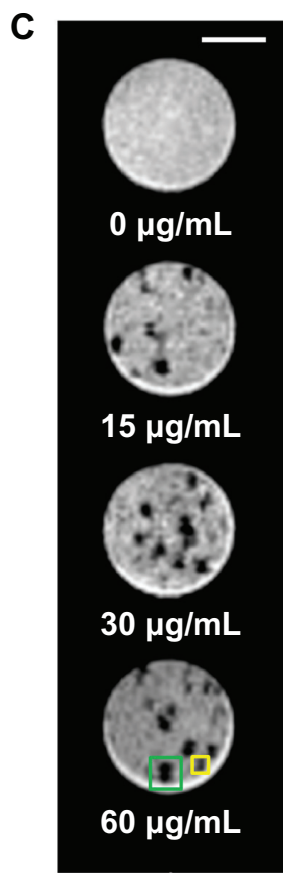

Figure 2 TEM, NMR spectroscopy and cellular MRI applied to NSCs treated with native as well as chitosan-USPIO NPs demonstrate chitosan-enhanced endocytic NP uptake. (A) Transmission electron micrographs of cells exposed to native NPs and chitosan-USPIO NPs. Without chitosan, few NPs appear within the cell; however, with chitosan, NPs appear within membranous vesicles within 2 hours of NP exposure at $15 \mu \mathrm{g} / \mathrm{mL}$ (scale bars $100 \mathrm{~nm}$ ). (B) NMR transverse relaxation measurements ( $\mathrm{n}=3$ ) reveal a loading dose dependence of chitosan-USPIO NP uptake from 0 to $30 \mu \mathrm{g} / \mathrm{mL}$, above which a saturation condition appears to dominate with little gain in uptake with increased loading dose. (C) $\mathrm{T}_{2}{ }^{*}$-weighted cellular MRI (I I.7 T) images of chitosan-USPIO NP-labeled single NSCs (yellow box) and small NSC clusters (neurospheres; green box) within 2 wt\% agarose-in-PBS gels.

Note: Cells are apparent at each concentration as localized regions of signal hypointensity (scale bar $500 \mu \mathrm{m})$.

Abbreviations: TEM, transmission electron microscopy; NMR, nuclear magnetic resonance; MRI, magnetic resonance imaging; NSCs, neural stem cells; USPIO NPs, ultrasmall superparamagnetic iron oxide nanoparticles; PBS, phosphate-buffered saline. 
that chitosan-USPIO NP uptake was achieved after incubation in as low as $7.5 \mu \mathrm{g} / \mathrm{mL}$ suspension of chitosan-USPIO NPs, with these nanoparticles visible exclusively within endosomes following uptake. In contrast, particles were not appreciably visible in cells treated with suspensions of native USPIO NPs, although they were visible in abundance outside of these cells close to the cell membrane (Figure 2A). In order to demonstrate the difference, the TEM sample treated with native USPIO NPs shown in Figure 2A was not subjected to washing steps for removal of NPs that were not uptaken by cells. Similarly, NMR relaxometry was utilized to measure the $\mathrm{T}_{2}$ of properly washed samples at various native and chitosan-modified USPIO NP concentrations. In these experiments, shorter $\mathrm{T}_{2}$ indicates a higher USPIO NP content and implies improved imaging contrast by $\mathrm{T}_{2}{ }^{*}$-weighted cellular MRI (Supplementary Figure 1). With NSCs, NMR spectroscopy indicated little appreciable uptake at any concentration of native NPs, consistent with TEM data. However, significant uptake of chitosan-modified USPIO NPs was observed, with the extent of nanoparticle uptake, within the range of $0-30 \mu \mathrm{g} / \mathrm{mL}$, proving strongly incubation dose dependent (Figure 2B). Above $30 \mu \mathrm{g} / \mathrm{mL}$, the additional increase in uptake of chitosan-modified USPIO NPs was severely diminished, likely indicating a surface saturation condition for the attaching nanoparticles. Finally, NSCs prepared similarly were seeded in a hydrated, soft, agarose gel and were imaged at high resolution using an 11.7 Tesla microimaging system with a $100-\mu \mathrm{m}$ slice thickness, $2.5-\mathrm{mm}$ field of view, and $39-\mu \mathrm{m}$ in-plane resolution. As shown in Figure 2C, individual NSCs and small NSC clusters were visible over a broad range of labeling concentrations, spanning $15-60 \mu \mathrm{g} / \mathrm{mL}$, providing proof of principle for chitosanUSPIO NP-enabled imaging at a resolution approaching the single-cell scale.

Furthermore, NSCs containing NPs were maintained under sterile culture conditions for 10 days and subjected to TEM and NMR relaxation studies over this period to evaluate NP retention. TEM showed that uptaken particles were initially contained within endocytic (membranous) vesicles (Figure 3A, left). However, between the day of uptake and day 10 post uptake, many particles achieved endosomal escape. These particles were observed within larger aggregates, free within the cytoplasm (Figure 3A, right) where they resided for several days after. This endosomal escape and corresponding long-term retention is supported by NMR relaxometry data (Figure 3B), offering an explanation for how particles remain in cells for many days beyond uptake without being rapidly trafficked through the endo-lysosomal pathways leading to exocytosis. For NMR relaxometry measurements, labeled
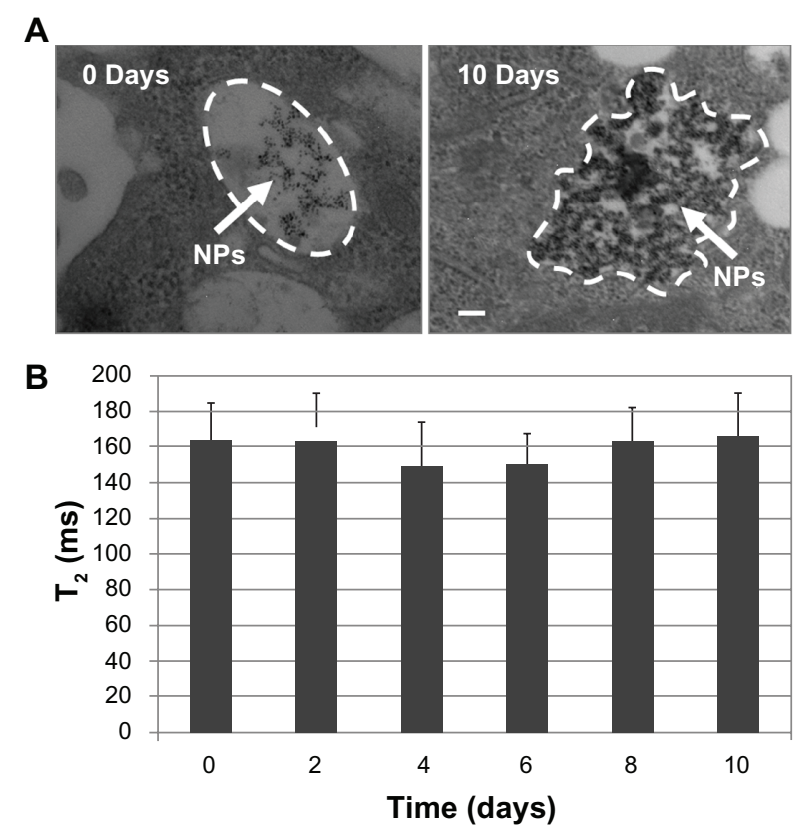

Figure 3 TEM and NMR relaxometry confirm endosomal escape of chitosanUSPIO NPs and their long-term retention by NSCs. (A) Transmission electron micrographs of cells containing chitosan-USPIO NPs on day 0 and 10 of culture. On day 0 , NPs are apparent within membranous vesicles within the cell; however, on day 10, NPs are found in irregularly shaped clusters within the cytosol (ie, outside of vesicles), appearing to have achieved endosomal escape, bypassing rapid lysosomal trafficking out of the cell (scale bar $50 \mathrm{~nm}$ ). (B) NMR relaxometry measurements ( $m e a n \pm S D, n=3$ ) of NSCs containing chitosan-USPIO NPs (loading dose $15 \mu \mathrm{g} / \mathrm{mL}$ ) taken at 2-day intervals confirm the presence of NPs at concentrations comparable to those just after loading over 10 days of culture, indicating NP retention for at least this period.

Abbreviations: TEM, transmission electron microscopy; NMR, nuclear magnetic resonance; USPIO NPs, ultrasmall superparamagnetic iron oxide nanoparticles; NSCs, neural stem cells.

NSCs were kept in culture until the day of measurement, trypsinized, centrifuged, and washed two times to remove potentially secreted NPs and then embedded in agarose gel in NMR tubes for T2 measurements as reported in Figure 3B.

In this context, endosomal escape is likely achieved due to a combination of increasing chitosan charge density within the endosome as acidification proceeds and also a reduction in endosome compartmental volume from membrane recycling. By calculation, using Katchalsky's equation relating chitosan amino-group $p K a,{ }^{27}$ environmental $p H$, and charge density $(1-\alpha)$ :

$$
p K a=p H+\log \left[\frac{1-\alpha}{\alpha}\right]
$$

an $85 \%$ deacytelated chitosan with $p K a=6.5$ realizes its highest charge density at roughly $\mathrm{pH} 5$ (Supplementary Figure 4), achieved in the late endosome, corresponding to the stage of smallest endosomal volume..$^{29,30}$ At the highest chitosan charge density and smallest endosomal compartmental 

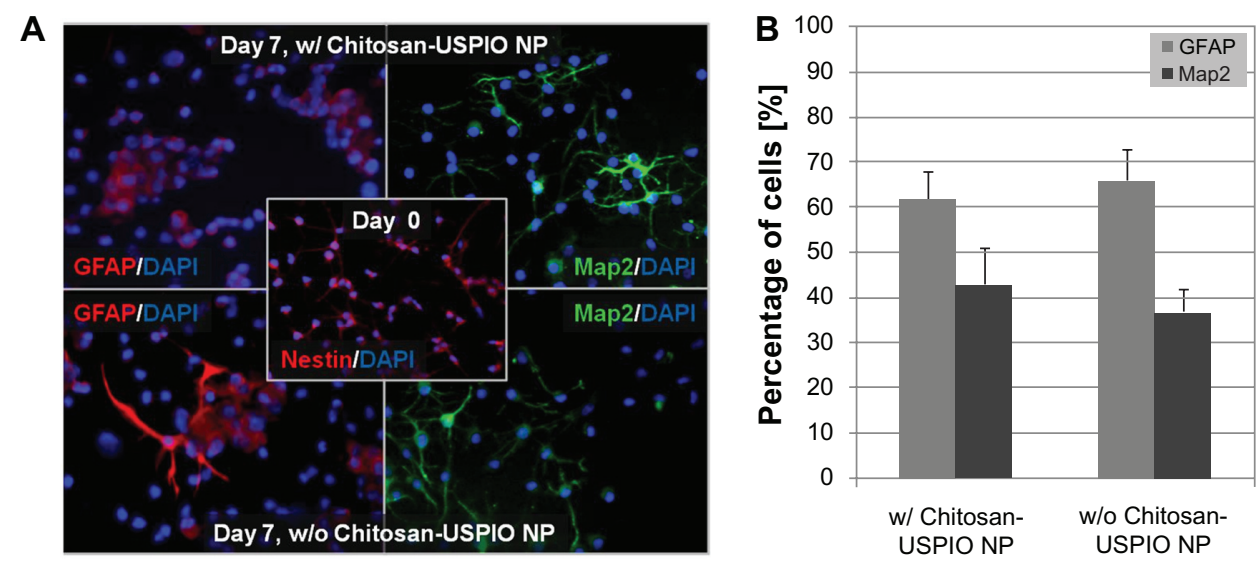

Figure 4 Indirect immunofluorescence staining of NSCs and NSC-derived cells for stem cell and differentiation markers (cells either labeled with I5 $\mu \mathrm{g} / \mathrm{mL}$ chitosan-USPIO NPs or unlabeled as controls). (A) NSC differentiation is unaffected by chitosan-USPIO labeling. Immunofluorescence staining reveals abundant cells positive for Nestin (NSC marker) on Day 0 and indistinguishable cell fractions positive for MAP2 (neuronal marker) or GFAP (astroglial marker) on Day 7 of NSC culture in differentiation media (NSC media, without growth factors, with fetal calf serum and retinoic acid). (B) Quantification of immunostaining for MAP2-and GFAP-positive cell fractions illustrates comparable results for NSCs with and without chitosan-USPIO NPs ( $n=3$ samples).

Abbreviations: NSCs, neural stem cells; USPIO NPs, ultrasmall superparamagnetic iron oxide nanoparticles.

volume, it is presumable that escape occurs during or before the late endosome stage by (1) membrane rupture due to internal charge repulsion and (2) osmotic swelling of the vesicle via the "proton sponge effect" within roughly 24 hours of uptake. ${ }^{30}$ Escaped particles have then avoided trafficking through lysosomal pathways and remain in the cytosol for at least 10 days (Figure 3B), enabling cell tracking over the same period.

After demonstrating efficient labeling of NSCs with these nanoparticles, cellular viability, expansion rate, and differentiation potential were assessed for NSCs exposed to our chitosan-USPIO NPs as well as for untreated control NSCs in order to evaluate if labeling with chitosan-USPIO NPs has any negative impact on NSC fate. Live/dead viability assay (CalceinAM/EtBr; Invitrogen) 12 hours, as well as 1 day and 10 days after loading (Supplementary Figure 5), revealed no quantifiable cytotoxicity for the chitosan-USPIO NPs. Proliferation rates were determined using WST-1 assays for NSCs labeled with $30 \mu \mathrm{g} / \mathrm{mL}$ chitosan-USPIO NPs and unlabeled NSCs. The proliferation rate for NSCs exposed to

A
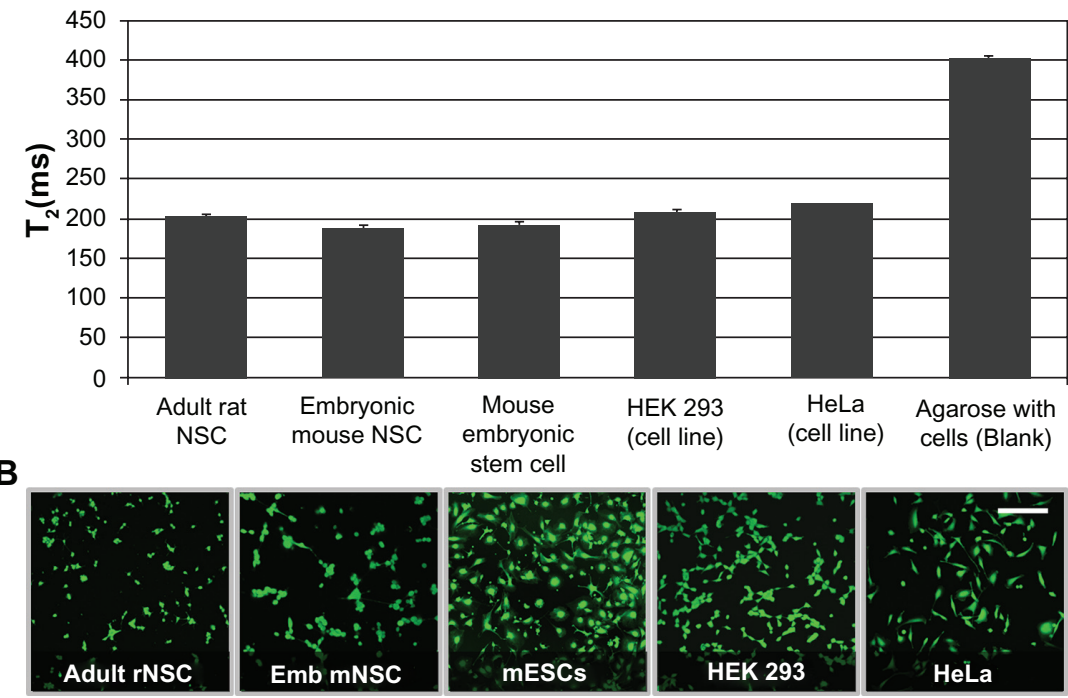

Figure $5 \mathrm{NMR}$ relaxometry measurements and live/dead (CalceinAM/EtBr) fluorescence staining of primary and immortalized cell types (all cells labeled with I 5 g//mL chitosanUSPIO NPs). (A) NMR relaxometry measurements of rat hippocampal NSCs, mouse cortical NSCs, mouse embryonic stem cells, HEK 293 kidney cells, and HeLa cervical cancer cells, 12 hours after loading and washing indicated comparably efficient labeling with NPs for each of the cell types. (B) Live/dead (CalceinAM/EtBr) staining of cells I 2 hours after loading showed no significant cytotoxic effect on cells, irrespective of cell type, with cells of each type exhibiting characteristic morphologies (scale bar $50 \mu \mathrm{m}$ ). Abbreviations: NMR, nuclear magnetic resonance; NSCs, neural stem cells; USPIO NPs, ultrasmall superparamagnetic iron oxide nanoparticles. 
chitosan-USPIO NPs was determined to be $24.7 \pm 3.1$ hours, which was not significantly different from the proliferation rate of unlabeled NSCs of $21.7 \pm 2.5$ hours $(n=3)$. Similarly, the differentiation potential (determined as fractions of MAP2+ neurons and GFAP+ glial cells by immunostaining) were comparable for cells treated with chitosan-USPIO NPs and untreated controls (Figure 4A and B). These data indicate that USPIO NP labeling does not negatively affect cellular proliferation or differentiation; however, additional future studies characterizing the genomic-level influence of these particles on labeled cells are warranted.

Finally, as our labeling technique does not involve any NSC-specific methodology, we tested the general applicability of this labeling strategy using a variety of primary and immortalized cell types. Adult rat hippocampal NSCs, mouse cortical NSCs, mouse embryonic stem cells, HEK 293 kidney cells, and HeLa cervical cancer cells were exposed to chitosan-USPIO NPs at $5 \times 10^{4}$ cells per cell type. All cells were labeled at a chitosan-USPIO NP concentration of $15 \mu \mathrm{g} / \mathrm{mL}$ for 2 hours, washed as described, and then transferred to 10-mm NMR tubes for relaxometry measurements or seeded in a 24-well plate for live/dead assessment. Based on measured T2 values (Figure 5A), NP loading efficiency was similar for each sample of each cell type, indicating broad applicability of our labeling scheme. Similarly, the live/dead viability assay 2 hours after loading revealed no quantifiable cytotoxicity for the chitosanUSPIO NPs, irrespective of cell type (Figure 5B). These data support chitosan functionalization as a compelling choice for efficient NP delivery to NSCs and also potentially a wide variety of other primary and immortalized cell types.

\section{Conclusion}

In conclusion, chitosan-USPIO NPs offer an efficient approach for magnetic labeling of cells, enabling cellular MRI-based tracking at the single-cell scale in vitro and potentially also in vivo over extended periods of time. TEM and NMR relaxometry confirmed endosomal chitosan-NP uptake, subsequent endosomal escape, and cytosolic retention by NSCs over a broad range of chitosan-NP loading concentrations. NSC viability and differentiation potential were unaffected by the labeling process, and cellular MRI demonstrated detection of single cells and small clusters of cells containing chitosan-NPs at close to single cell resolution. Additionally, a variety of primary and immortalized cell types were similarly labeled with comparable high efficiencies, with each sample exhibiting high viability and cellular morphologies characteristic of the cell line. These data support the notion that chitosan-USPIO NPs may be used as a general approach to efficiently magnetically label cells, with tremendous promise for development and evaluation of cellular therapies in in vitro tissue models as well as animal models of human disease.

\section{Acknowledgments}

The authors thank the Molecular Biosensors and Imaging Center for access to cell culture facilities, the Philip and Marsha Dowd Seed Fund, Pittsburgh Life Sciences Greenhouse, the Pittsburgh NMR Center (NIH P41 EB001977), and the National Science Foundation (CAREER Award: 0748062) for support.

\section{Disclosure}

The authors report no conflicts of interest in this work.

\section{References}

1. Doetsch F, Caille I, Lim DA, Garcia-Verdugo JM, Alvarez-Buylla A. Subventricular zone astrocytes are neural stem cells in the adult mammalian brain. Cell. 1999;97(6):703-716.

2. Gage FH. Mammalian neural stem cells. Science. 2000;287(5457): $1433-1438$.

3. Lois C, Garcia-Verdugo JM, Alvarez-Buylla A. Chain migration of neuronal precursors. Science. 1996;271(5251):978-981.

4. Reynolds BA, Weiss S. Generation of neurons and astrocytes from isolated cells of the adult mammalian central nervous system. Science. 1992;255(5052):1707-1710.

5. Palmer TD, Takahashi J, Gage FH. The adult rat hippocampus contains primordial neural stem cells. Mol Cell Neurosci. 1997;8(6):389-404.

6. Eriksson PS, Perfilieva E, Bjork-Eriksson T, et al. Neurogenesis in the adult human hippocampus. Nat Med. 1998;4(11):1313-1317.

7. Pluchino S, Quattrini A, Brambilla E, et al. Injection of adult neurospheres induces recovery in a chronic model of multiple sclerosis. Nature. 2003;422(6933):688-694.

8. Yandava BD, Billinghurst LL, Snyder EY. "Global" cell replacement is feasible via neural stem cell transplantation: evidence from the dysmyelinated shiverer mouse brain. Proc Natl Acad Sci U S A. 1999;96(12):7029-7034.

9. Bjorklund A, Lindvall O. Cell replacement therapies for central nervous system disorders. Nat Neurosci. 2000;3(6):537-544.

10. Bjugstad KB, Teng YD, Redmond DE Jr, et al. Human neural stem cells migrate along the nigrostriatal pathway in a primate model of Parkinson's disease. Exp Neurol. 2008;211(2):362-369.

11. Park KI, Teng YD, Snyder EY. The injured brain interacts reciprocally with neural stem cells supported by scaffolds to reconstitute lost tissue. Nat Biotechnol. 2002;20(11):1111-1117.

12. Teng YD, Lavik EB, Qu X, et al. Functional recovery following traumatic spinal cord injury mediated by a unique polymer scaffold seeded with neural stem cells. Proc Natl Acad Sci U S A. 2002;99(5):3024-3029.

13. Pastori C, Librizzi L, Breschi GL, et al. Arterially perfused neurospherederived cells distribute outside the ischemic core in a model of transient focal ischemia and reperfusion in vitro. PLOS ONE. 2008;3(7):e2754.

14. Bjornerud A, Johansson L. The utility of superparamagnetic contrast agents in MRI: theoretical consideration and applications in the cardiovascular system. NMR Biomed. 2004;17(7):465-477.

15. Wu YL, Ye Q, Foley LM, et al. In situ labeling of immune cells with iron oxide particles: an approach to detect organ rejection by cellular MRI. Proc Natl Acad Sci U S A. 2006;103(6):1852-1857.

16. Dodd SJ, Williams M, Suhan JP, Williams DS, Koretsky AP, Ho C. Detection of single mammalian cells by high-resolution magnetic resonance imaging. Biophys J. 1999;76(1 Pt 1):103-109. 
17. Kanno S, Lee PC, Dodd SJ, Williams M, Griffith BP, Ho C. A novel approach with magnetic resonance imaging used for the detection of lung allograft rejection. J Thorac Cardiovasc Surg. 2000;120(5):923-934.

18. Ye Q, Yang D, Williams M, et al. In vivo detection of acute rat renal allograft rejection by MRI with USPIO particles. Kidney Int. 2002;61(3):1124-1135.

19. Arbab AS, Yocum GT, Wilson LB, et al. Comparison of transfection agents in forming complexes with ferumoxides, cell labeling efficiency, and cellular viability. Mol Imaging. 2004;3(1):24-32.

20. Janjic JM, Srinivas M, Kadayakkara DK, Ahrens ET. Self-delivering nanoemulsions for dual fluorine-19 MRI and fluorescence detection. J Am Chem Soc. 2008;130(9):2832-2841.

21. Chakraborty SK, Fitzpatrick JA, Phillippi JA, et al. Cholera toxin B conjugated quantum dots for live cell labeling. Nano Lett. 2007;7(9): 2618-2626.

22. Shah BS, Clark PA, Moioli EK, Stroscio MA, Mao JJ. Labeling of mesenchymal stem cells by bioconjugated quantum dots. Nano Lett. 2007;7(10):3071-3079.

23. Ruoslahti E. RGD and other recognition sequences for integrins. Annu Rev Cell Dev Biol. 1996;12:697-715.
24. Roy K, Mao HQ, Huang SK, Leong KW. Oral gene delivery with chitosan - DNA nanoparticles generates immunologic protection in a murine model of peanut allergy. Nat Med. 1999;5(4):387-391.

25. Mao HQ, Roy K, Troung-Le VL, et al. Chitosan-DNA nanoparticles as gene carriers: synthesis, characterization and transfection efficiency. J Control Release. 2001;70(3):399-421.

26. Corsi K, Chellat F, Yahia L, Fernandes JC. Mesenchymal stem cells, MG63 and HEK293 transfection using chitosan-DNA nanoparticles. Biomaterials. 2003;24(7):1255-1264.

27. Strand SP, Tommeraas K, Varum KM, Ostgaard K. Electrophoretic light scattering studies of chitosans with different degrees of N-acetylation. Biomacromolecules. 2001;2(4):1310-1314.

28. Sorlier P, Denuziere A, Viton C, Domard A. Relation between the degree of acetylation and the electrostatic properties of chitin and chitosan Biomacromolecules. 2001;2(3):765-772.

29. Murphy RF. Maturation models for endosome and lysosome biogenesis. Trends Cell Biol. 1991;1(4):77-82.

30. Storrie B. Endosomes and Lysosomes: A dynamic relationship, 1. In: Storrie BaM RF, editor. Elsevier; 1993:2-16. 


\section{Supplementary figures}

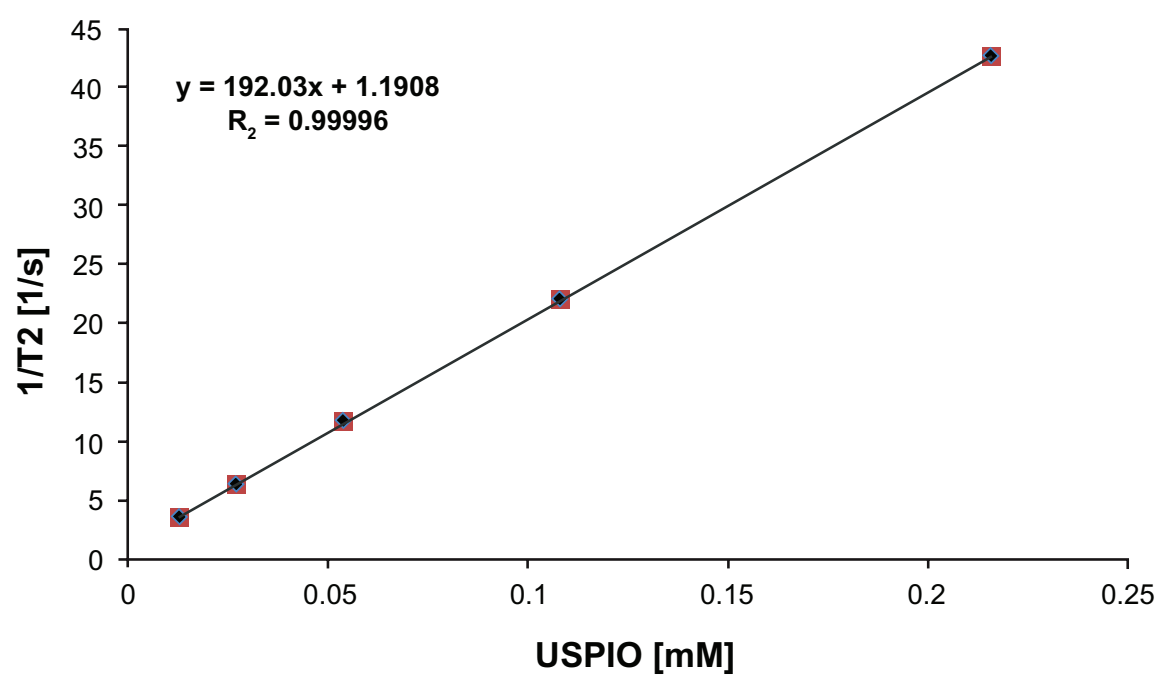

Figure SI Chitosan-USPIO NPs exhibit superior $\mathrm{T}_{2}$ relaxivity $\left(192.0 \mathrm{mM}^{-1}(\mathrm{Fe}) \mathrm{s}^{-1}\right)$ from 0 to $0.22 \mathrm{mM} \mathrm{Fe}$.

A

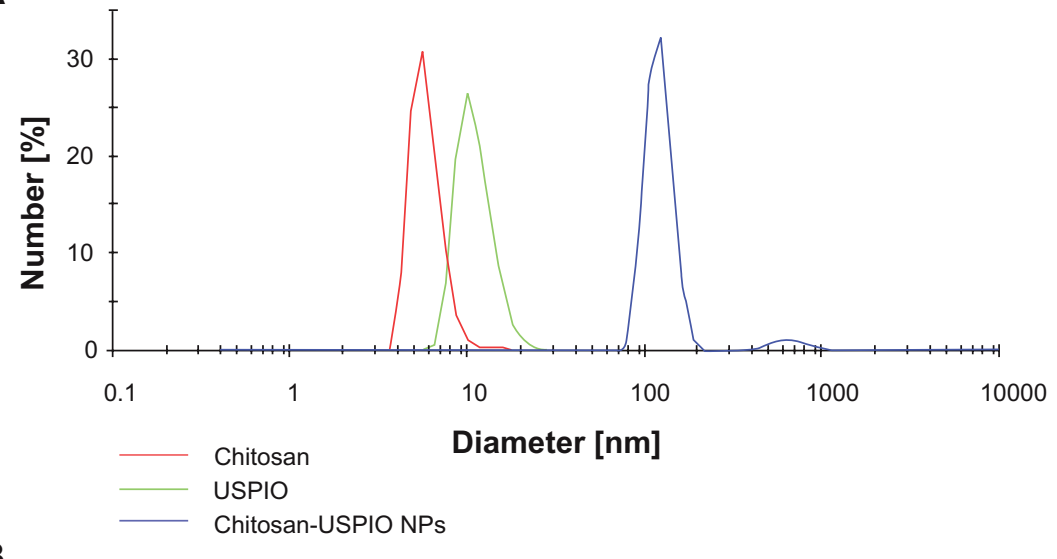

B

\begin{tabular}{|l|c|c|c|}
\hline & Chitosan & USPIO & Chitosan-USPIO \\
\hline Mean diameter [nm] & 10.1 & 5.6 & 122.4 \\
\hline Zeta potential [nm] & 19.6 & -21.9 & 4.2 \\
\hline
\end{tabular}

Figure S2 Dynamic light scattering and zeta potential measurements (Zetasizer Nano ZS, Malvern Instruments) for chitosan, USPIO NPs, and chitosan-USPIO NPs. (A) Apparent diameter distributions of NPs and NP constituents are measured in PBS, at concentrations presented in materials and methods for chitosan-USPIO NP synthesis. (B) Mean apparent diameter and zeta potential for chitosan, USPIO NPs, and chitosan-USPIO NPs show an increase in apparent NP size after chitosan conjugation and show a significant change to a positive value for zeta potential. 


\section{A}
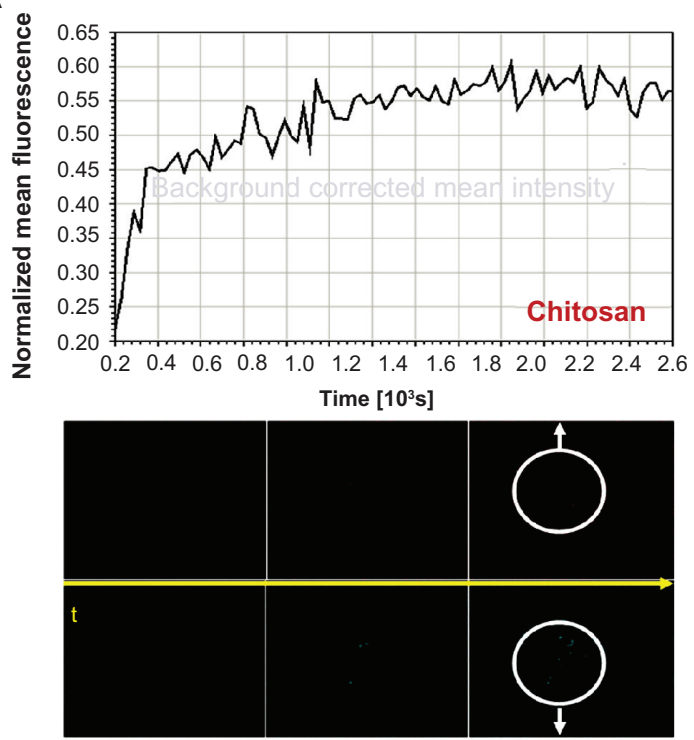

B

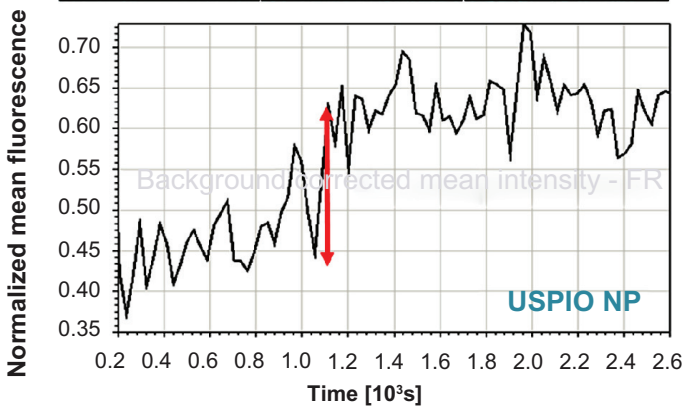

Figure S3 Mean fluorescence intensity of a confocal optical section for chitosan (red) and NP (green) fluorescence channels over time.

Notes: Chitosan mean fluorescence intensity changes markedly over small time scales, indicating fluorescent parcels entering and leaving the plane of imaging (ie, nanoparticles in membranous vesicles). Chitosan fluorescence intensity does not display such variability over small time scales, suggesting that chitosan is distributed more uniformly, also outside of vesicles, detaching and escaping with time from the NP surface (scale bar $10 \mu \mathrm{m}$ ).

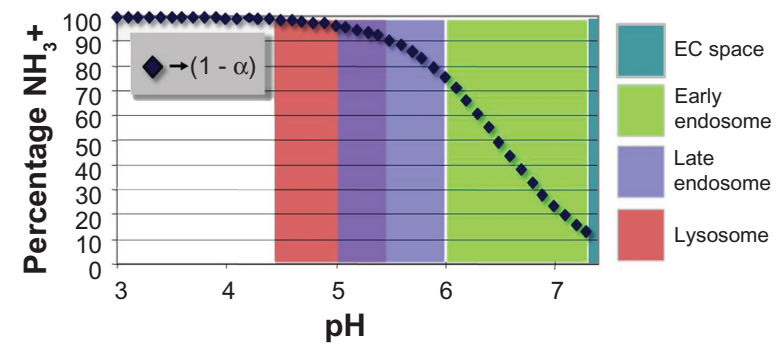

Figure S4 Increase in chitosan charge with decreasing $\mathrm{pH}$.

Note: Fraction of charged amino groups $(\mathrm{I}-\alpha)$ on $85 \%$ deacytelated chitosan with $p K a=6.5$, by Katchalsky equation.

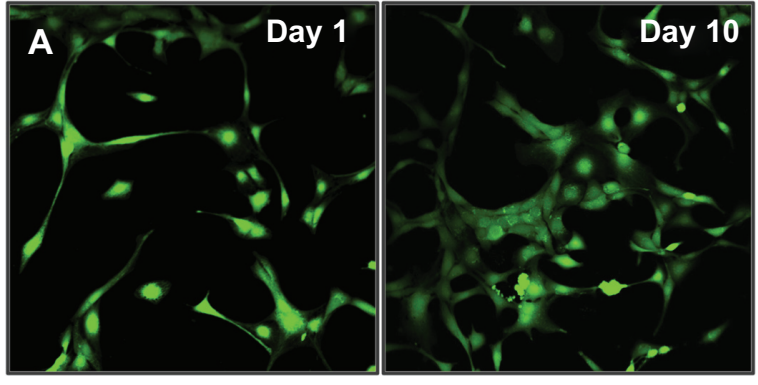

B

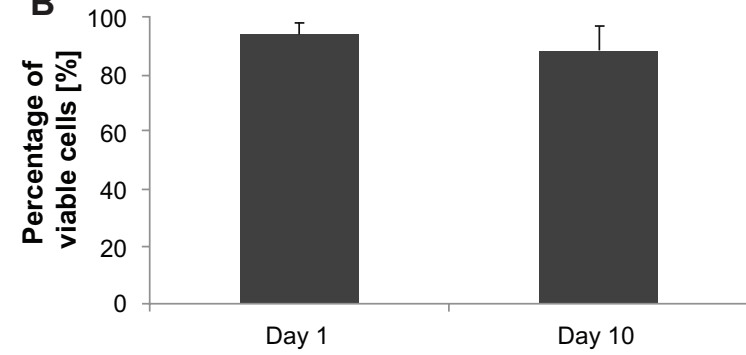

Figure S5 Live/dead ${ }^{\circledR}$ cell viability assay for NSCs after treatment with chitosanUPSIO NPs on Day I (24 hours after treatment) and Day 10. (A) Calcein-AM staining for live cells confirms a typical, adherent, NSC morphology on each day. (B) Quantification of living cell fraction was performed on Day I and Day 10 and revealed a high viable cell fraction with no significant difference between time points.
International Journal of Nanomedicine

\section{Publish your work in this journal}

The International Journal of Nanomedicine is an international, peerreviewed journal focusing on the application of nanotechnology in diagnostics, therapeutics, and drug delivery systems throughou the biomedical field. This journal is indexed on PubMed Central, MedLine, CAS, SciSearch ${ }^{\circledR}$, Current Contents ${ }^{\circledR} /$ Clinical Medicine,

\section{Dovepress}

Journal Citation Reports/Science Edition, EMBase, Scopus and the Elsevier Bibliographic databases. The manuscript management system is completely online and includes a very quick and fair peer-review system, which is all easy to use. Visit http://www.dovepress.com/ testimonials.php to read real quotes from published authors.

Submit your manuscript here: http://www.dovepress.com/international-journal-of-nanomedicine-journal 